\title{
Bound state for dielectric waveguide with high contrast inset in the core
}

\author{
Maria Faleeva* , Igor Popov \\ ITMO University, Department of Higher Mathematics, 197101 St. Petersburg, Russia
}

\begin{abstract}
We consider cylindrical dielectric waveguide with a core having a high contrast inset. It is assumed, that for certain conditions, for a waveguide with an insertion in the core there is a bound state appears below the bottom of the continuous spectrum. In this paper we pose the problem of proving the existence of such state and obtain an upper estimate of the distance from the eigenvalue to the threshold.
\end{abstract}

\section{Introduction}

Optical fibers containing inclusions with high contrast in permittivity are widely used (see, e.g., [1-5]). We consider a cylindrical dielectric waveguide (permittivity $\varepsilon=\varepsilon_{1}$ ) with a core (permittivity $\varepsilon=\varepsilon_{2} \gg \varepsilon_{1}$ ). The core has an insertion with width $2 a$ of a material higher contrast. The waveguide is unbounded in the direction of z. We show that a bound state below the bottom of the continuous spectrum exists for the system. Variational approach is used. Propagation of electromagnetic waves along the waveguide is described by a scalar dimensionless Helmholtz equation for the electric field. For proof we need to construct the trial function, approximately satisfying the equation, and obtain the necessary estimate, which should be negative. The method is analogous to that suggested in [6]. This method is applicable to eigenvalues below the continuous spectrum. The result can be generalized to the periodic system of such insets in the core. In this case, one has an additional spectral band instead of the bound state. It is possible that such periodic system has photonic crystal property. As for eigenvalues lying in spectral gaps, the method should be essentially modified [7]. There are numerous works concerning to eigenvalue below the threshold for various waveguides systems (see, e.g., [8] and references therein). Trapped modes (i.e. eigenvalues embedded in the continuous spectrum) are of special interest. There is an extensive literature about the occurrence of trapped modes in curved closed waveguides (see, e.g., $[9,10])$.

\section{The method of the proof}

It is assumed that we deal with monochromatic light passes along the axis. Analysis of electromagnetic waves reduces to the investigation of the scalar equation for the electric field. Mode solutions for waveguide are obtained from the dimensionless Helmholtz equation for (harmonic dependence on time $\mathrm{t}\left(e^{i \omega t}\right)$ is taken into account),

$$
\frac{\partial^{2} E_{z}}{\partial r^{2}}+\frac{1}{r} \frac{\partial E_{z}}{\partial r}+\frac{1}{r^{2}} \frac{\partial^{2} E_{z}}{\partial \varphi^{2}}+\frac{\partial^{2} E_{z}}{\partial z^{2}}+k^{2} E_{z}=0,
$$

where $k=d \omega \sqrt{\varepsilon \mu}, \mathrm{d}$ is the radius of the waveguide. Multiply the radius of the waveguide on the wave number allows you to get the dimensionless equation, and all the variables having the dimensions of length, normalized to the $\mathrm{d}$.

We take $E_{z}=\Psi(r, z) e^{i(v \varphi+\omega t)}$. Then eigenfunction $\Psi$ satisfies the equation

$$
-\frac{\partial^{2} \Psi}{\partial r^{2}}-\frac{1}{r} \frac{\partial \Psi}{\partial r}-\frac{\partial^{2} \Psi}{\partial z^{2}}+\frac{v^{2}}{r^{2}} \Psi=k^{2} \Psi
$$

and can be constructed using $\Psi_{1}, \Psi_{2}$, and $\Psi_{3}$, where

$$
\Psi=\left\{\begin{array}{c}
\Psi_{1}, r \in[0, \delta] \\
\Psi_{2}, r \in[\delta, 1] . \\
\Psi_{3}, r \in[1, \infty]
\end{array} .\right.
$$

The left side of the equation is the action of the operator $\mathrm{H}$, is the operator with the matching conditions at the interfaces $r=\delta$ and $r=1$ :

$$
\begin{aligned}
& H \Psi=k^{2} \Psi \\
& \left.\Psi_{3}\right|_{(r=1)}=\left.\Psi_{2}\right|_{(r=1)} \\
& \left.\Psi_{1}\right|_{(r=\delta)}=\left.\Psi_{2}\right|_{(r=\delta)} \\
& \left.\frac{d \Psi_{3}}{d r}\right|_{(r=1)}=\left.\frac{d \Psi_{2}}{d r}\right|_{(r=1)} \\
& \left.\frac{d \Psi_{1}}{d r}\right|_{(r=\delta)}=\left.\frac{d \Psi_{2}}{d r}\right|_{(r=\delta)}
\end{aligned}
$$

In accordance with the variational principles, minimum of the ratio

$$
\frac{(H \Psi, \Psi)-\left\|\Psi \sqrt{k^{2}-\beta^{2}}\right\|^{2}}{\|\Psi\|^{2}},
$$


is reached if is an eigenfunction of the operator $H$, it equals to the distance from the eigenvalue to the threshold. If there exists a trial function $\Psi$ (satisfying the matching conditions at the interfaces) such that the function is negative:

$$
M(\Psi)=(H \Psi, \Psi)-\left\|\Psi \sqrt{k^{2}-\beta^{2}}\right\|^{2}<0,
$$

then there exists an eigenvalue $\Lambda^{2}$ less than the threshold, $\Lambda^{2} \leq \lambda^{2}=k^{2}-\beta^{2}$. Thus, the idea is to find a trial function, which makes the expression $M(\Psi)$ negative.

We take the trial function $\Psi$ as $\Psi=F+G$, where $F=\alpha u(r) f(z), G=\eta s(r) g(z)$,

$$
\mathrm{u}(r)=\left\{\begin{array}{l}
N_{1} J_{v}\left(\lambda_{1} r\right)+M_{4} J_{v}\left(\lambda_{4} r\right), r \in[0, \delta] \\
N_{2} J_{v}\left(\lambda_{2} r\right)+M_{2} N_{v}\left(\lambda_{2} r\right), r \in[\delta, 1], \\
N_{3} \mathrm{~K}_{v}(\gamma r), r \in[1, \infty]
\end{array}\right.
$$

$J_{v}, N_{v}, K_{v}$ are Bessel functions, $\lambda_{i}^{2}=k_{i}^{2}-\beta^{2}, k_{i}^{2}=$ $\omega^{2} \varepsilon_{i} \mu_{0} d^{2}, \lambda_{3}=i \gamma, \gamma^{2}=\beta^{2}-k_{2}^{2}$,

$$
\begin{gathered}
s(r)=\left\{\begin{array}{l}
e^{-\frac{\pi r}{2 a}}, r \in\left[0, \frac{1}{2}\right] \\
2(1-r) e^{-\frac{\pi}{4 a}}, r \in\left[\frac{1}{2}, 1\right], \\
0, r \in[0, \infty]
\end{array}\right. \\
f(z)=\min \left\{\begin{array}{l}
\left.1, e^{-q(|z|-a)}\right\}=\left\{\begin{array}{l}
1, z \in[-a, a] \\
e^{-q(|z|-a)}, z \in[-\infty,-a] \cup[a, \infty]
\end{array}\right. \\
g(z)=\left\{\begin{array}{l}
\cos \left(\frac{\pi z}{2 a}\right), z \in[-a, a] \\
e^{-q(|z|-a)}, z \in[-\infty,-a] \cup[a, \infty]
\end{array}\right.
\end{array}\right.
\end{gathered}
$$

where $\alpha, \eta, q$ are free parameters of the trial function, $\mathrm{d}$ is the radius of the waveguide, $\delta$ is the radius of the core $(0 \leq \delta \ll 1), 2 \mathrm{a}$ is a width of the inset in the core $(0 \leq a \ll 1)$.

The functions $f(z)$ and $g(z)$ are z symmetrical.

$$
f(-\infty)=f(\infty)=0, \mathrm{~g}(-\infty)=g(\infty)=0 .
$$

Functions $\mathrm{u}(r)$ and $s(r)$ satisfy the condition:

$$
\int_{0}^{\infty} r u(r) d r=\int_{0}^{\infty} r s(r) d r=0
$$

Lemma 1: The trial function $\Psi$ defined in (8)-(11) satisfies the following condition:

$$
(H \Psi, \Psi)=\left\|\Psi_{r}\right\|^{2}+\left\|\Psi_{z}\right\|^{2}+\int_{-\infty}^{\infty} \int_{-\infty}^{\infty} \frac{v^{2}}{r} \Psi^{2} d r d z
$$

Proof:

$$
\begin{aligned}
& (H \Psi, \Psi)=\int_{-\infty}^{\infty} \int_{-\infty}^{\infty} r\left(-\frac{\partial^{2} \Psi}{\partial r^{2}}-\frac{1}{r} \frac{\partial \Psi}{\partial r}-\frac{\partial^{2} \Psi}{\partial z^{2}}+\frac{v^{2}}{r^{2}} \Psi\right) \Psi d r d z= \\
& =-\int_{-\infty}^{\infty} \int_{-\infty}^{\infty}\left(\frac{\partial}{\partial r}\left(r \frac{\partial \Psi}{\partial r}\right)+r \frac{\partial^{2} \Psi}{\partial z^{2}}-\frac{v^{2}}{r} \Psi\right) \Psi d r d z
\end{aligned}
$$

$(H \Psi, \Psi)=-\left.\int_{-\infty}^{\infty}\left(r \frac{\partial \Psi}{\partial r} \Psi\right)\right|_{0} ^{\infty} d z+\int_{-\infty}^{\infty} \int_{0}^{\infty} r\left(\frac{\partial \Psi}{\partial r}\right)^{2} d r d z$

$-\left.\int_{0}^{\infty} r\left(\frac{\partial \Psi}{\partial z} \Psi\right)\right|_{-\infty} ^{\infty} d r+\int_{0}^{\infty} r \int_{-\infty}^{\infty}\left(\frac{\partial \Psi}{\partial z}\right)^{2} d z+\int_{-\infty}^{\infty} \int_{0}^{\infty} \frac{v^{2}}{r} \Psi^{2} d r d z$

Then, from conditions (12)-(13), one has:

$(H \Psi, \Psi)=\int_{-\infty}^{\infty} \int_{0}^{\infty} r\left(\frac{\partial \Psi}{\partial r}\right)^{2} d r d z+\int_{0}^{\infty} r \int_{-\infty}^{\infty}\left(\frac{\partial \Psi}{\partial z}\right)^{2} d z d r$

$+\int_{-\infty}^{\infty} \int_{0}^{\infty} \frac{v^{2}}{r} \Psi^{2} d r d z=\left\|\Psi_{r}\right\|^{2}+\left\|\Psi_{z}\right\|^{2}+\int_{-\infty}^{\infty} \int_{0}^{\infty} \frac{v^{2}}{r} \Psi^{2} d r d z$

Lemma 2: The form $M(\Psi)$ for the trial function $\Psi$ defined in (8)-(11) can be represented as follows:

$$
M(\Psi)=\left\|F_{z}\right\|^{2}+\left\|G_{z}\right\|^{2}+2 \int_{-\infty}^{\infty} \int_{0}^{\infty} r F_{z} G_{z} d r d z
$$

Proof:

$M(\Psi)=\left\|\Psi_{r}\right\|^{2}+\left\|\Psi_{z}\right\|^{2}+\int_{-\infty}^{\infty} \int_{-\infty}^{\infty} \frac{v^{2}}{r} \Psi^{2} d r d z-\left\|\Psi \sqrt{k^{2}-\beta^{2}}\right\|^{2}=$

$=\int_{-\infty}^{\infty} \int_{0}^{\infty} r\left(\frac{\partial(F+G)}{\partial r}\right)^{2} d r d z+\int_{0}^{\infty} r \int_{-\infty}^{\infty}\left(\frac{\partial(F+G)}{\partial z}\right)^{2} d z d r-$

$-\int_{-\infty}^{\infty} \int_{-\infty}^{\infty} r\left(k^{2}-\beta^{2}-\frac{v^{2}}{r^{2}}\right)(F+G)^{2} d r d z$

We take some integrals by parts and after some calculations, one has

$$
\begin{aligned}
& M(\Psi)=-\int_{-\infty}^{\infty} \int_{0}^{\infty} r F\left(\left(F_{r r}+G_{r r}\right)+\frac{1}{r}\left(F_{r}+G_{r}\right)\right) d r d z- \\
& -\int_{-\infty}^{\infty} \int_{0}^{\infty} r F(F+G)\left(k^{2}-\beta^{2}-\frac{v^{2}}{r^{2}}\right) d r d z- \\
& -\int_{-\infty}^{\infty} \int_{0}^{\infty} r G\left(\left(F_{r r}+G_{r r}\right)+\frac{1}{r}\left(F_{r}+G_{r}\right)\right) d r d z+ \\
& +\int_{-\infty}^{\infty} \int_{0}^{\infty} r G\left((F+G)\left(k^{2}-\beta^{2}-\frac{v^{2}}{r^{2}}\right)\right) d r d z+ \\
& +\int_{-\infty}^{\infty} \int_{-\infty}^{\infty}\left(r F_{z}^{2}+r G_{z}^{2}+2 r F_{z} G_{z}\right) d r d z
\end{aligned}
$$

The first and the second integrals in the previous formula are equal to zero and we obtain our lemma.

Next, we need to estimate the resulting expression. Write out its as:

$$
\begin{aligned}
& M(\Psi)=\alpha^{2} \int_{-\infty}^{\infty}\left(\frac{d f(z)}{d z}\right)^{2} d z \int_{0}^{\infty} r(\mathrm{u}(r))^{2} d r+\eta^{2} \int_{-\infty}^{\infty}\left(\frac{d g(z)}{d z}\right)^{2} d z \\
& \int_{0}^{\infty} r(\mathrm{~s}(r))^{2} d r+2 \alpha \eta \int_{-\infty}^{\infty} \frac{d f(z)}{d z} \frac{d g(z)}{d z} d z \int_{0}^{\infty} r u(r) \mathrm{s}(r) d r
\end{aligned}
$$

The square of the norm of the trial function has the form:

Using integration by parts, one can write: 


$$
\begin{aligned}
& \|\Psi\|^{2}=\alpha^{2} \int_{-\infty}^{\infty}(f(z))^{2} d z \int_{0}^{\infty} r(\mathrm{u}(r))^{2} d r+\eta^{2} \int_{-\infty}^{\infty}(g(z))^{2} d z \\
& \int_{0}^{\infty} r(\mathrm{~s}(r))^{2} d r+2 \alpha \eta \int_{-\infty}^{\infty} \mathrm{a}(z) g(z) d z \int_{0}^{\infty} r u(r) \mathrm{s}(r) d r
\end{aligned}
$$

Integrals of the functions $f(z)$ and $g(z)$ can be calculated. To estimate the integrals containing Bessel functions use their asymptotic representations:

$$
\begin{aligned}
& J_{v}(\lambda r) \approx \sqrt{\frac{2}{\pi \lambda r}} \cos \left(\lambda r-\frac{v \pi}{2}-\frac{\pi}{4}\right) \\
& N_{v}(\lambda r) \approx \sqrt{\frac{2}{\pi \lambda r}} \sin \left(\lambda r-\frac{v \pi}{2}-\frac{\pi}{4}\right) \\
& \mathrm{K}_{v}(\gamma r) \approx \sqrt{\frac{\pi}{2 \gamma r}} e^{-\gamma r}
\end{aligned}
$$

For the trial function $\Psi$ defined in (8)-(11) the estimation of the ratio has the form:

$$
\begin{aligned}
& \frac{\mathrm{M}(\Psi)}{\|\Psi\|^{2}} \leq\left(\frac{2 \alpha^{2} q}{\pi} A+q B+\eta^{2}\left(\frac{a}{4}+\frac{q a^{2}}{\pi^{2}}\right) C\right) \\
& \left(\frac{2 \alpha^{2}}{\pi}\left(2 a+\frac{1}{q}\right) A+\frac{4 a}{\pi} B+\frac{\eta^{2} a^{3}}{\pi^{2}} C\right)^{-1},
\end{aligned}
$$

where

$$
A=\left(\left(\frac{N_{1}}{\sqrt{\lambda_{1}}}+\frac{M_{4}}{\sqrt{\lambda_{4}}}\right)^{2} \delta+\frac{\left(N_{2}+M_{2}\right)^{2}}{\lambda_{2}}(1-\delta)+\frac{\pi^{2} N_{3}^{2}}{4 \gamma^{2}}\right),-
$$$$
B=\frac{4}{3} \sqrt{\frac{2}{\pi}} \alpha \eta\left(\left(\frac{N_{1}}{\sqrt{\lambda_{1}}}+\frac{M_{4}}{\sqrt{\lambda_{4}}}\right) \delta^{\frac{3}{2}}+\frac{\left(N_{2}+M_{2}\right)}{\sqrt{\lambda_{2}}}\left(2^{-\frac{3}{2}}-\delta^{\frac{3}{2}}+\right.\right.
$$$$
\left.\left.+2 e^{-\frac{\pi}{4 a}} \frac{(8 \sqrt{2}-7)}{20 \sqrt{2}}\right)\right) \text {, }
$$$$
C=\left(e^{-\frac{\pi}{2 a}}\left(\frac{5 \pi^{2}}{48 a^{2}}-\frac{\pi}{2 a}-1\right)+1\right) \text {. }
$$

We take $N_{3}=1$. The coefficients $\mathrm{N}_{2}, M_{2}, N_{1}, M_{4}$ are obtained from the system of boundary conditions:

$$
\left\{\begin{array}{l}
N_{1} J_{v}\left(\lambda_{1} \delta\right)+M_{4} J_{v}\left(\lambda_{4} \delta\right)=N_{2} J_{v}\left(\lambda_{2} \delta\right)+M_{2} N_{v}\left(\lambda_{2} \delta\right), \\
N_{2} J_{v}\left(\lambda_{2}\right)+M_{2} N_{v}\left(\lambda_{2}\right)=K_{v}(\gamma), \\
\left.N_{1}\left(\frac{d}{d r} J_{v}\left(\lambda_{1} r\right)\right)\right|_{r=\delta}+\left.M_{4}\left(\frac{d}{d r} J_{v}\left(\lambda_{4} r\right)\right)\right|_{r=\delta}= \\
=\left.N_{2}\left(\frac{d}{d r} J_{v}\left(\lambda_{2} r\right)\right)\right|_{r=\delta}+\left.M_{2}\left(\frac{d}{d r} N_{v}\left(\lambda_{2} r\right)\right)\right|_{r=\delta}, \\
\left.N_{2}\left(\frac{d}{d r} J_{v}\left(\lambda_{2} r\right)\right)\right|_{r=1}+\left.M_{2}\left(\frac{d}{d r} N_{v}\left(\lambda_{2} r\right)\right)\right|_{r=1}= \\
=\left.\left(\frac{d}{d r} K_{v}(\gamma r)\right)\right|_{r=1} \cdot
\end{array}\right.
$$

This coefficients obtained numerically for some values of the parameters of the trial function and for fixed dependencies of geometrical characteristics of the waveguide. The results are summarized in Table 1 .

The tables show that there are many different combinations of parameter sets the trial function for which there is a value of the permittivity of the inset in the core of the waveguide that the ratio $\mathrm{M}(\Psi) /\|\Psi\|^{2}$ will be negative. This means that there is a spectral point below the threshold of the continuous spectrum.

\section{Conclusions}

Thus, we show that for a cylindrical dielectric waveguide having a core with a high contrast inset, there is a bound state below the bottom of the continuous spectrum and we obtain an upper estimate of the distance from the eigenvalue to the threshold. This result allows us to assume that in the case of the periodic system of inserts in the core, system can obey the photonic crystal property which can find many applications.

This work was partially financially supported by the Government of the Russian Federation (grant 074-U01), by Ministry of Science and Education of the Russian Federation (GOSZADANIE 2014/190, Projects No 14.Z50.31.0031 and No. $1.754 .2014 / \mathrm{K})$ ), by grants MK-5001.2015.1, MK5161.2016.1 of the President of the Russian Federation, by grant 16-11-10330 of Russian Science Foundation

\section{References}

1. S.G. Johnson, J.D. Joannopoulos, Photonic Crystals, the Road from Theory to Practice (Boston, Kluwer, 2002)

2. W. Axmann, P. Kuchment, L. Kunyansky, J. Light. Tech., 17, 1996-2007 (1999)

3. A. Tip, A. Moroz, J.M. Combes, J. Phys. A: Math. Gen., 33, 6223-6252 (2000)

4. I.Y. Popov, A.I. Trifanov, E.S. Trifanova, Comp. Math Math. Phys., 50(11), 1830-1836 (2010)

5. A.D. Kiselev, D.O. Plutenko, Nanosystems: Physics, Chemistry, Mathematics, 7(2), 349-370 (2016)

6. P. Exner, S. Vugalter, Ann. Inst. Henri Poincare, 65, 109-123 (1996)

7. Langer, M., Strauss, M., 2016, Triple variational principles for self-adjoint operator functions, J. Func. Anal., Vol. 270, pp. 2019-2047

8. Linton, C. M., McIver, P., 2007, Embedded trapped modes in water waves and acoustics, Wave Motion, Vol. 45, pp. 16-29.

9. Dauge, M., Raymond, N., 2012, Plane waveguides with corners in the small angle limit, J.Math. Phys., Vol. 53, p. 123529.

10. Duclos, P., Exner, P., 1995, Curvature-induced bound states in quantum waveguides in two and three dimensions, Rev. Math. Phys., Vol. 7, pp. 73-102. 
Table 1. Parameters of the trial function which give negative values of the ratio

\begin{tabular}{|c|c|c|c|c|c|c|c|c|c|c|}
\hline № & $\alpha$ & $\eta$ & $v$ & $\mathrm{q}$ & $\lambda_{1}$ & $\lambda_{2}$ & $\lambda_{4}$ & $\gamma$ & $\mathrm{a}$ & $\delta(\mathrm{a})$ \\
\hline 1 & 0.001 & 1000 & 2 & 1 & 50 & 10 & 26 & 0.1 & 0.01 & $a^{0.5}$ \\
\hline 2 & 0.001 & 1000 & 2 & 1 & 50 & 10 & 46 & 0.1 & 0.01 & $a^{0.1}$ \\
\hline 3 & 0.01 & 1000 & 2 & 1 & 100 & 1 & 82 & 1 & 0.001 & $a^{0.01}$ \\
\hline 4 & 0.001 & 1000 & 2 & 1 & 10 & 1 & 130 & 0.1 & 0.001 & $a^{0.01}$ \\
\hline 5 & 0.5 & 500 & 0 & 1 & 100 & 1 & 202 & 0.1 & 0.001 & $a^{0.01}$ \\
\hline 6 & 0.01 & 100 & 0 & 10 & 100 & 1 & 162 & 0.1 & 0.001 & $a^{0.01}$ \\
\hline
\end{tabular}

